\title{
Assessment of accuracy of immediate blood separation method: a novel blood analysis strategy
}

\author{
Kunio Nakayama $\cdot$ Kanehisa Morimoto
}

Received: 3 April 2009/Accepted: 4 May 2010/Published online: 9 June 2010

(C) The Japanese Society for Hygiene 2010

\begin{abstract}
Objectives This study assesses the accuracy of the immediate blood separation method, a novel blood sampling strategy that enables blood analysis in any possible location.

Methods We conducted a cross-validation study between data from immediate blood separation and conventional methods. During the annual medical examinations in 2006 of a company located in an Osaka suburb, blood was drawn from workers ( $n=256$; males 200 , females 56 ) by puncturing their middle finger as well as venipuncture of the antecubital vein, by medical personnel. The following nine parameters were evaluated by autoanalyzer: aspartate aminotransferase (AST), alanine aminotransferase (ALT), $\gamma$-glutamyl transpeptidase $(\gamma \mathrm{GT})$, triglyceride, total cholesterol, high-density lipoprotein (HDL) cholesterol, urea nitrogen, uric acid, and creatinine.

Results After comparing data from the two methods using correlation analysis and regression analysis, we found a close $R^{2}$ value (coefficient of determination) relationship that ranged from 0.996 to 1.000 for each item. The $R^{2}$ value was 0.998 for $\log$ AST, 0.997 for Log ALT, 0.999 for $\log \gamma \mathrm{GT}, 1.000$ for Log triglyceride, 1.000 for total cholesterol, 0.999 for HDL cholesterol, 0.998 for urea nitrogen, 0.999 for uric acid, and 0.996 for creatinine. Relationship was satisfactory for all nine items tested.

Conclusion Our results prove the reliability of data from the immediate blood separation method in an occupational health setting. The method enables self-testing by medically
\end{abstract}

K. Nakayama $\cdot$ K. Morimoto $(\square)$ Department of Social and Environmental Medicine, Osaka University Graduate School of Medicine F1, 2-2 Yamada-oka, Suita, Osaka 565-0871, Japan e-mail: morimoto@envi.med.osaka-u.ac.jp unskilled people, which is an important process to prevent lifestyle-related diseases.

Keywords Immediate blood separation method ·

Self-testing · Occupational health .

Lifestyle-related diseases · Micro-analysis

\section{Introduction}

As Japan's population ages, preventing lifestyle-related diseases and promoting health are issues of growing concern. Promotion of health is strongly associated with modification of lifestyle factors [1]. Checking medical data is a crucial process for increasing motivation to alter lifestyle factors. A simple, quick, and accurate medical data system that can be conducted by nonmedical personnel is crucial to prevent onset and exacerbation of lifestyle-related diseases, especially diabetes mellitus $[2,3]$.

Several self-testing methods have already been developed [2-4]. Since one important technological problem of self-testing was the blood separation process, new blood sampling methods have been developed. The immediate blood separation method (Leisure, Tokyo) enables blood self-sampling (self-drawing), immediate blood separation, and transport by posting the sample for analysis of health indicators [5]. The device enables analysis of health indicators by taking blood samples in every possible location and testing the obtained plasma at laboratories. However, few reports have assessed its accuracy in clinical, occupational health, and community health settings.

To analyze the accuracy of the immediate blood separation method, we simultaneously performed blood tests by immediate blood separation and conventional methods (blood sampling by venipuncture of the antecubital vein 
and blood cell separation by centrifugation) during medical examinations in an occupational health setting. Both samples were analyzed for biochemical parameters concerning lifestyle-related diseases. We assessed the reliability of the data from both methods.

\section{Materials and methods}

\section{Study population}

The subjects ( $n=321$; males 245 , females 76) of this study were employees of a hard metal tool manufacturing corporation in an Osaka suburb who were receiving their annual medical examination at their workplace in July 2006. In Japan, an annual medical examination for employees includes blood examination for prevention of lifestyle-related diseases according to occupational safety and health regulations [6]. At this corporation, the blood examination of an annual health check includes such items as aspartate aminotransferase (AST), alanine aminotransferase (ALT), $\gamma$ glutamyl transpeptidase ( $\gamma \mathrm{GT})$, alkaline phosphatase (ALP), zinc sulfate turbidity test (ZTT), total protein (TP), triglyceride, total cholesterol, high-density lipoprotein (HDL) cholesterol, urea nitrogen, creatinine, glucose, uric acid, white blood cells, red blood cells, hemoglobin, hematocrit, and platelets. All employees of this company had blood drawn by venipuncture of the antecubital vein by medical personnel at every annual medical examination. At the 2006 medical examination, workers had blood drawn by puncturing their middle finger as well as by venipuncture of the antecubital vein by medical personnel. Some workers preferred venipuncture of the antecubital vein. Of the 321 workers, 256 workers (males 200, females 56) had blood drawn by both methods. The mean age and standard deviation (SD) of the subjects was $44.2 \pm 10.7$ (19-63) years for males and $43.2 \pm 11.7$ (23-60) years for females.

\section{Ethics approval}

The study including analysis of blood samples from these subjects was approved by the Ethics Committee for Osaka University Graduate School of Medicine (approval no. 482). We conducted this survey in connection with the approved survey, as medical practice of industrial physician. All participants gave their written informed consent.

Method

\section{Components of the immediate blood separation method}

The components of the immediate blood separation method included a capillary, a tube, a cylinder, and a piston cap
(Fig. 1). The capillary is a loaded sponge device that absorbs $50 \mu \mathrm{l}$ blood (Fig. 1, 1). The tube contains $450 \mu \mathrm{l}$ dilution buffer that does not permeate the red corpuscle membrane due to an ingeniously converted 4-(2-hydroxyethyl)-1-piperazineethanesulfonic acid (HEPES) buffer (Fig. 1, 2). The cylinder, which contains a filtering membrane comprised of a five-layer membrane consisting of membrane and glass-fiber filters, is constructed so that no corpuscles remain in the plasma layer (Fig. 1, 3). The piston cap seals the tube (Fig. 1,4). The reliability of this method was proven by laboratory data. Horita et al. [7] reported evaluations of the utility of this system with excellent correlation coefficients of 1.000 for AST, 1.000 for ALT, 0.997 for $\gamma \mathrm{GT}, 0.997$ for triglyceride, 0.995 for HDL cholesterol, 0.989 for low-density lipoprotein (LDL) cholesterol, 0.996 for urea nitrogen, 0.980 for creatinine, and 0.995 for uric acid. These device vessels have been recognized by patents in Japan [8-10], the USA [11], and the European Union (EU) [12]. This blood sampling kit has been approved as an authorized medical device in Japan (nos. 21600BZZ00007000 and 21700BZZ00020000).

\section{Blood sampling and plasma skimming of the immediate blood separation method}

The blood sampling and plasma skimming procedures of the immediate blood separation method are shown in Fig. 2. In this investigation, blood sampling and plasma skimming processes were conducted by medical stuff to ensure precise procedures. Participants' middle finger was sterilized (Fig. 2, 1) then punctured by lancet (Fig. 2, 2), and blood was drawn. Fifty microliters of blood was absorbed by capillary using the sponge device (Fig. 2, 3 and 4). The sponge device was then dropped into a tube containing $450 \mu \mathrm{l}$ dilution buffer (Fig. 2, 5). After mixed with the dilution buffer (Fig. 2, 6), it was slowly pushed vertically into a cylinder with a filtering membrane and then into a cylinder for separating the drawn blood into

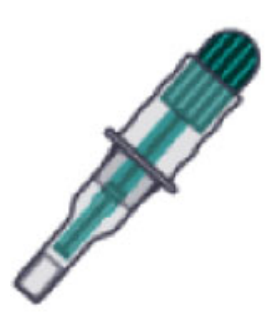

1

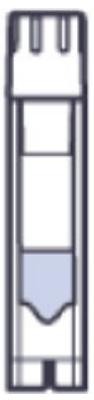

2

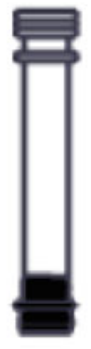

3

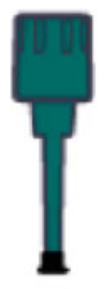

4
Fig. 1 Components of the immediate blood separation device: 1 capillary with sponge device, 2 tube containing $450 \mu 1$ dilution buffer, 3 cylinder with filtering membrane comprised of five-layer membrane consisting of membrane and glass-fiber filters, 4 piston cap 

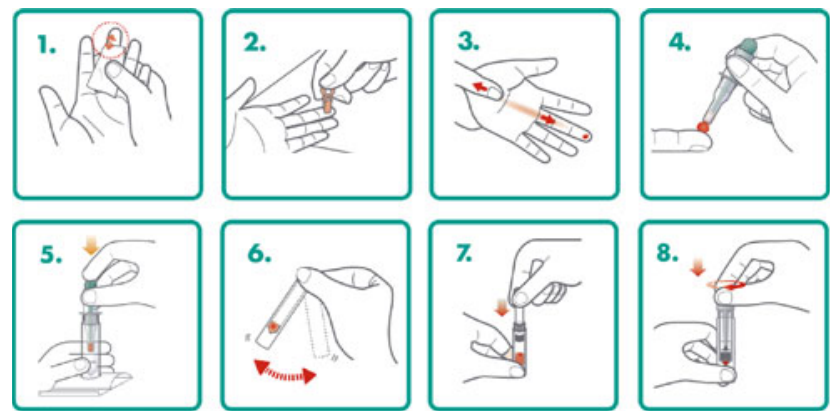

Fig. 2 Blood sampling and immediate blood separation method: 1 sterilization, 2 string to tip of middle finger, 3 bleeding, 4 absorbing $50 \mu \mathrm{l}$ blood by capillary with sponge device, 5 sponge device dropped into tube containing dilution buffer, 6 mixing with dilution buffer, 7 diluted blood separated into corpuscles and plasma by cylinder with filtering membrane, 8 sealing by piston cap

corpuscles and plasma (Fig. 2, 7). This blood separation process needs only a few seconds. An important factor is the precise filtering membrane, which is comprised of five layers consisting of both membrane and glass-fiber filters [4]. The cylinder was sealed by a piston cap and mailed to a laboratory for analysis (Fig. 2, 8). The entire immediate blood separation method process from finger sterilization (Fig. 2, 1) to sealing by the piston cap (Fig. 2, 8) needs only $2-3$ min, even if conducted by a medically unskilled person.

\section{Conventional blood sampling method}

The conventional blood sampling method was conducted as follows: blood sampling was performed by venipuncture of the antecubital vein, $10 \mathrm{ml}$ blood was absorbed through a cylinder, and blood cell separation was performed by centrifugation at $3000 \mathrm{rpm}$ for $25 \mathrm{~min}$.

\section{Blood analysis}

Nine parameters (AST, ALT, $\gamma \mathrm{GT}$, triglyceride, total cholesterol, HDL cholesterol, urea nitrogen, uric acid, and creatinine) were evaluated by autoanalyzer BM-2250 (Nihon Denshi, Japan) from blood samples of both the immediate blood separation and conventional methods. The following formula was used to measure the diluted plasma from the samples of the immediate blood separation method [5]:

Measurement $($ device value $)=$ dilution value $\times$ DF (dilution factor)

$\times$ revision factor

$\mathrm{DF}=E_{1} /\left(E_{1}-E_{2}\right)$

$E_{1} \quad$ absorbance of marker substance in HEPES buffer in cylinder
$E_{2}$

absorbance of marker substance in HEPS buffer with diluted blood in cylinder

Revision factor correction coefficient for precise measurement defined in each measurement item

Both blood sampling methods, i.e., immediate blood separation and conventional, employed routine blood drawing techniques. The nine blood tests we analyzed were routine items for occupational health settings in Japan. For these reasons, the processes of this study were within the limits of routine occupational health work. After randomization of the sample number of blood samples, they were analyzed by autoanalyzer BM-2250 (Nihon Denshi, Japan). We conducted data matching and assessed the accuracy of the data.

Statistic analysis

Data were analyzed using Statistical Package for Social Science software version 14.0 (SPSS, Chicago, IL) for Windows. We studied cross-validation between the immediate blood separation method and conventional method by using Pearson's correlation analysis and linear regression analysis with least-squares method.

\section{Results}

Table 1 presents the Pearson's correlation coefficients between the values of the samples from the immediate blood separation method and venipuncture of the antecubital vein for the nine items. When comparing the data from the immediate blood separation and conventional methods by Pearson's correlation analysis, we found a close relationship with the following correlation coefficients: $0.93(p<0.001)$ for Log AST, $0.94(p<0.001)$ for Log ALT, $0.99(p<0.001)$ for Log $\gamma \mathrm{GT}, 0.99(p<0.001)$ for Log triglyceride, $0.99(p<0.001)$ for total cholesterol, $0.99(p<0.001)$ for HDL cholesterol, $0.98(p<0.001)$ for urea nitrogen, $0.99(p<0.001)$ for uric acid, and 0.93 $(p<0.001)$ for creatinine.

When comparing the data using linear regression analysis with least-squares method, we found a close relationship, with $R^{2}$ (coefficient of determination) values that ranged from 0.996 to 1.000 . The following $R^{2}$ values were indicated: 0.998 for Log AST, 0.997 for Log ALT, 0.999 for $\log \gamma \mathrm{GT}, 1.000$ for Log triglyceride, 1.000 for total cholesterol, 0.999 for HDL cholesterol, 0.998 for urea nitrogen, 0.999 for uric acid, and 0.996 for creatinine. The following regression formulas were indicated: $y=1.01 x$ for $\log$ AST, $y=1.00 x$ for $\log$ ALT, $y=1.01 x$ for $\log \gamma \mathrm{GT}, y=1.01 x$ for Log triglyceride, $y=0.98 x$ for 
Table 1 Correlation between values of immediate blood separation device and conventional method

\begin{tabular}{lll}
\hline & $\begin{array}{l}\text { Correlation } \\
\text { coefficients }\end{array}$ & $p$ \\
\hline Log AST & 0.93 & $<0.001$ \\
Log ALT & 0.94 & $<0.001$ \\
Log $\gamma$ GT & 0.99 & $<0.001$ \\
Log triglyceride & 0.99 & $<0.001$ \\
Total cholesterol & 0.99 & $<0.001$ \\
HDL cholesterol & 0.99 & $<0.001$ \\
Urea nitrogen & 0.98 & $<0.001$ \\
Uric acid & 0.99 & $<0.001$ \\
Creatinine & 0.93 & $<0.001$ \\
\hline
\end{tabular}

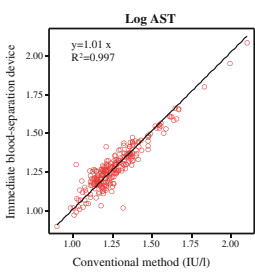

Log Triglyceride

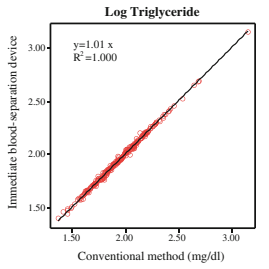

Urea nitrogen
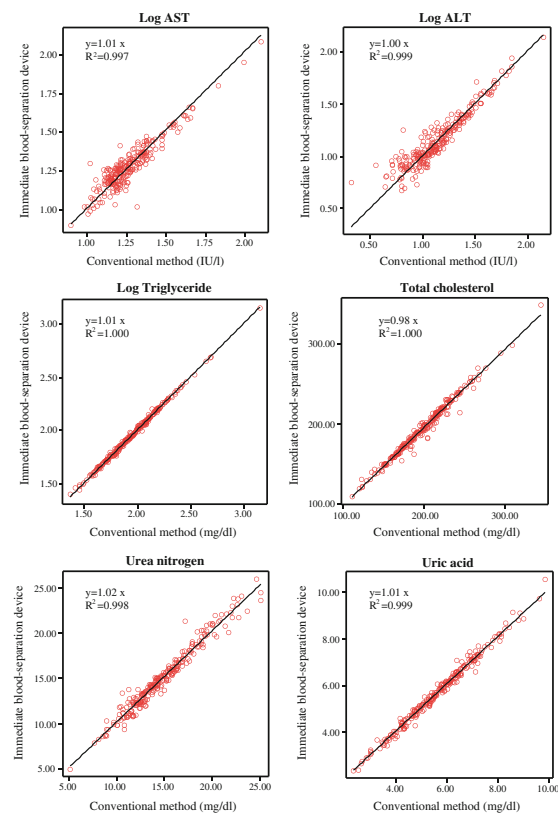

Total cholesterol

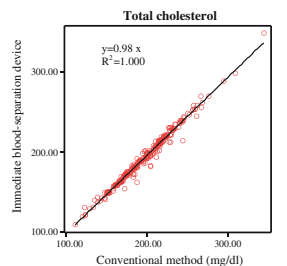

Uric acid
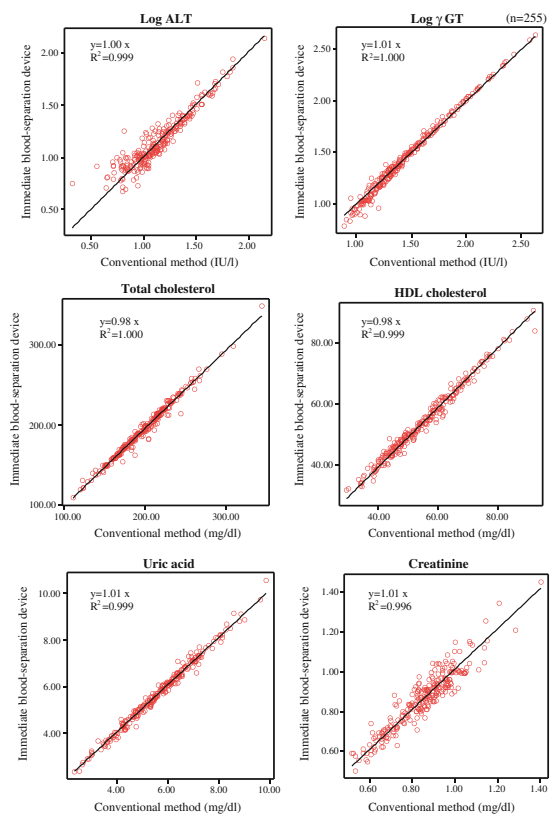

Fig. 3 Relationships between values of immediate blood separation device and conventional method: linear regression analysis with leastsquares method. Immediate blood separation device: blood sampling, string to tip of middle finger, absorbing $50 \mu \mathrm{l}$ blood, blood cell separation using immediate blood separation device, measuring by autoanalyzer BM-2250 (Nihon Denshi, Japan). Conventional method: blood sampling, venipuncture of antecubital vein, absorption of $10 \mathrm{ml}$ blood, blood cell separation by centrifugation at $3000 \mathrm{rpm}$ for 25 min, measuring by autoanalyzer BM-2250 (Nihon Denshi, Japan)

total cholesterol, $y=0.98 x$ for HDL cholesterol, $y=1.02 x$ for urea nitrogen, $y=1.01 x$ for uric acid, and $y=1.01 x$ for creatinine ( $x$, measurement value of conventional method; $y$, measurement value of immediate blood separation method). For all items, linearity was satisfactory, as indicated in Fig. 3.

\section{Discussion}

Self-tests, which can be used to diagnose conditions without involving a doctor, nurse, or other health professional [4, 5], might increase motivation for lifestyle modification to prevent lifestyle-related diseases. In Japan, several self-testing methods have been used as mailing tests or over-the-counter (OTC) tests [4].

Many self-testing methods for blood have also been reported, including a paper filter method, a hand-operated centrifugation plasma separator method [13, 14], and membrane plasma-skimming method. The problems with these self-sampling methods are difficulty of operation and sample preservation [15]. The paper filter method's blood sampling technique was easy to operate for medically unskilled people, but its preservation state was inadequate because the plasma-skimming process was not included [4]; analysis of items was limited in the paper filter method [4]. On the other hand, the hand-operated centrifugation plasma separator method newly enabled a good state of preservation, because the blood separation process, which was included in the centrifugation process, was applied $[15,16]$. However, the centrifugation process created difficulties for medically unskilled persons. The membrane plasma-skimming method, which uses a special plasmaskimming filter, was easy enough to be done by everybody. The immediate blood separation method might be easier than other methods.

The process for precise self-testing includes the blood drawing and blood separation processes [17]. The blood drawing process was performed by lancet puncture, which everybody recognized as very easy. In the blood separation process, the characteristic features of the filtering membrane that caused adsorption of substances also caused a "fudge factor" in data analysis. To correct the data for these errors in the immediate blood separation method, revision factors defined for each measurement item were applied [4]. The secondary problem with the blood separation process was hemolysis. In this method, an ingeniously converted HEPES buffer, which does not permeate red corpuscle membranes, and a precise filtering membrane, comprised of five layers consisting of both membrane and glass-fiber filters, was constructed to prevent contamination of the plasma layer by corpuscles [4]. These devices protect the plasma from the effect of hemolysis and enable ultramicro blood analysis using this method [14].

One limitation of this study was that the immediate blood separation method was performed by medical personnel as part of a company's annual medical examination. This might increase the data accuracy compared with data gathered by nonmedical personnel. However, the process of the immediate blood separation method was easy enough to enable such medically unskilled persons to 
produce diluted plasma from a single drop of capillary blood. For these reasons, the significant relationship indicated in this study might also be recognized when using the same method based on self-testing conducted by nonmedical personnel. Further investigation to analyze the accuracy of this method when conducted by medically unskilled persons is important.

Based on the results of our study, the data indicated significant relationship for all items (Fig. 3). We found a close relationship in correlation analysis for all nine tested items, with $R^{2}$ values ranging from 0.996 to 1.000 . The immediate blood separation method was proven to be a reliable alternative for venous blood sampling for the nine measurement items tested. In principle, this method's effectiveness can be extended to almost all blood analysis items. The immediate blood separation method was recognized as an effective procedure in situations where blood sampling is difficult, including occupational health, homes, schools, rural areas, and with children and the mentally impaired, for example.

\section{Conclusions}

Our results suggest that data from the immediate blood separation method are reliable in occupational health settings. We found a close relationship for AST, ALT, $\gamma$ GT, triglyceride, total cholesterol, HDL cholesterol, urea nitrogen, uric acid, and creatinine.

Acknowledgments We thank Leisure Co. Tokyo for sampling and analyzing data by the immediate blood separation method. No other support was received. We also thank Dr. Lu (Osaka Univ.) for ethical procedure, Dr. Hamaguchi (Leisure Co.) for scientific advice, and Dr. Inoguchi (Leisure Co.) for blood analysis of the nine items tested.

Conflict of interest statement We declare that we have no competing interests.

\section{References}

1. Morimoto K, editor. Lifestyle and health-health theory and demonstrable research. Tokyo: Igakusyoin; 1991.
2. Yum SI, Roe J. Capillary blood sampling for self-monitoring of blood glucose. Diabetes Technol Ther. 1999;1:39-40.

3. Suzuki Y. Painless blood sampling for self blood glucose measurement. Lancet. 1992;339:816-7.

4. Urata T, Sakurabayashi I. Current status for OTC examination (test). Rinsho Byori. 2005;53:1129-40.

5. Maehata E, Shiba T, Yano M, Horita M, Koga O, Arai K. Assessment of ultramicro device assay for diabetic patients. Proceeding of 18th congress of the international diabetes federation. Diabetes Metab. 2003;29:4S192.

6. Japan's e-Government. Act database homepage. Occupational safety and health regulation. Ministry of Internal Affairs and Communications of Japan. 2009. http://law.e-gov.go.jp/htmldata/ S47/S47HO057.html. Accessed 2 Oct 2009.

7. Horita M, Sugimoto S, Hokazono E, Osawa S. Establishment of mail medical examination system using immediate plasma separating device by the self-collection blood-the method of dilution ratio calculation by using internal standard for the sample with different amount of collecting blood. Rinsho Byori. 2008;56:577-83.

8. Arai T, Nomura N. Implement and method for separation of living-body sample. JP Patent 3597827, 25 Sept 2003.

9. Arai T, Nomura N. Biochemical analytical method, its device and biochemical analytical cartridge. JP Patent 3445791, 3 Dec 2003.

10. Nanba H, Koga O, Hotta M, Ota Y. Biological sample-preparing method, biological sample-quantitative determination method, and biological sample-preserving container. JP Patent 3698696, 6 June 2003.

11. Nanba H, Koga O, Horita M, Ohta Y. Method of preparing biological samples for quantification. U.S. Patent 6,936,473, 30 Aug 2005.

12. Nanba H, Horita M, Koga O, Ohta Y. Instrument and method for blood separation, and preparing method, quantifying method and preserving container for biological sample. E.U. Patent EP1221614, 10 July 2002.

13. Ryan A, Greenfield S, Wilson S. Prevalence and determinants of the use of self-tests by members of the public: a mixed methods study. BMC Public Health. 2006;25:193.

14. Ryan A, Wilson S, Greenfield S, Clifford S, McManus RJ, Pattison HM. Range of self-tests available to buy in the United Kingdom: an Internet survey. J Public Health. 2006;28:370-4.

15. Kamaura M, Ohono H, Imai K, Kawakami C, Okamoto N, Tochikubo O. A novel method for the self-collection of blood, "thenar lancing phlebotomy" and investigation of its accuracy. Rinsho Byori. 2006;54:671-8.

16. Kamaura M, Ohono H, Takahashi M, Takahashi T, Tochikubo O. Evaluation of a guidance method for reducing lifestyle-related diseases by the self-monitoring of blood collection in small- and medium-sized enterprises. Sangyo Eiseigaku Zasshi. 2007;49:89-97.

17. Higashino K. Sampling and conveyance of mail testing. Rinsho Byori. 2005;53:1122-8. 